\title{
Anxiety and mood disorder in young males with mitral valve prolapse
}

\author{
For-Wey Lung ${ }^{1-4}$ \\ Chih-Tao Cheng ${ }^{5}$ \\ Wei-To Chang ${ }^{6}$ \\ Bih-Ching Shu ${ }^{7}$
}

'Department of Psychiatry, Kaohsiung Armed Forces General Hospital, Kaohsiung, Taiwan; ${ }^{2}$ Graduate Institute of Behavioral Sciences, Kaohsiung Medical Center, Taiwan; ${ }^{3}$ Department of Psychiatry, National Defense Medical Center, Taipei, Taiwan; ${ }^{4}$ Calo Psychiatric Center, Pingtung County, Taiwan; ${ }^{5}$ School of public Health, University of California, Berkeley, CA, USA; ${ }^{\circ} \mathrm{Liu}$ Chia-Hsiu Hospital, Kaohsiung County, Taiwan; ${ }^{7}$ Institute of Allied Health Sciences and Department of Nursing, National Cheng Kung University, Tainan, Taiwan
Correspondence: Bih-Ching Shu Institute of Allied Health Sciences and Department of Nursing, National Cheng Kung University, No I Da-Hsueh Rd., Tainan, Taiwan, 701

Tel + $88662353535 \# 5822$

Fax +8866273750

Email forweylung@gmail.com
Objective: This study explored the prevalence of panic disorder and other psychiatric disorders in young Han Chinese males with mitral valve prolapse (MVP). With the factors of age, sex, and ethnicity controlled, the specific role of MVP in panic disorder was analyzed.

Methods: Subjects with chest pain aged between 18 and 25 years were assessed with the echocardiograph for MVP and the Chinese version of the Mini-International Neuropsychiatric Interview for panic disorder $(\mathrm{n}=39)$.

Results: Of the 39 participants, $35.9 \%$ met the diagnosis of anxiety disorder, $46.2 \%$ met at least one criterion of anxiety disorder, and $23.1 \%$ met the diagnostic criteria of major depressive disorder. There was no statistically significant difference in the prevalence of panic disorder between one of the (8.3\%) MVP patients, and two (7.4\%) control participants.

Conclusions: There is a high prevalence of psychiatric disorder, including anxiety disorder and major depressive disorder, in those who report pain symptoms, so that diagnosis and treatment of these patients is of great importance. In addition, individuals with MVP did not have an increased risk for panic disorder. Whether MVP may be a modifier or mediating factor for panic disorder needs to be further assessed in a larger scale study.

Keywords: mitral valve prolapse, panic disorder, Han Chinese males, major depressive disorder, echocardiograph, MINI

\section{Introduction}

There is a high cormorbidity between the psychiatric symptoms of depression and anxiety, and somatoform disorders in primary care (Mergl et al 2007). Patients in primary care who report pain symptoms are often found to have depression or anxiety, but these psychiatric disorders are underdiagnosed (Means-Christensen et al 2008). One study found that only half of the depressed patients were recognized, and only $15 \%$ to $26 \%$ were diagnosed (Lecrubier 2007). Another study found that $19.5 \%$ of patients in primary care had anxiety disorder, but nearly half of these patients remained untreated (Kroenke et al 2007; Weisberg et al 2007).

The patient's distress increases if he or she cannot be correctly diagnosed, and thus receive adequate treatment or if, after correct diagnosis appropriate treatment is not available in a primary care setting. Such events may mean higher utilization of medical care services (Demertzis and Craske 2006). However, effective psychotherapeutic intervention for primary care patients has been developed (Lang et al 2006). Thus understanding of these medically unexplained somatic symptoms and psychiatric comorbidities is important to help physicians provide more suitable diagnoses and treatment for these patients (Schur et al 2007).

Among these psychiatric disorders, panic disorder has been estimated to have $4 \%$ to $6 \%$ prevalence in the general population (Messias et al 2007). The diagnosis of panic disorder, as first listed in the DSM-III, biologically differentiated it from anxiety neurosis (Lieb 2005). Predisposing factors for panic disorder include a genetic 
contribution (Freitag et al 2006; Fyer et al 2006; Rothe et al 2006), which has been found to have ethnic heterogeneity (Domschke et al 2007), and gender specificity (Unschuld et al 2008). Compared with males, females have almost twice the risk for developing panic disorder (Andrade et al 1996). Panic disorder has been found to be highly comorbid with agoraphobia (Bienvenu et al 2006) and migraine (Swartz et al 2000), and patients who have co-morbid disorders have a greater severity of panic disorder (Andrade et al 1996). Precipitating factors which increase the risk of panic disorder include a family history of panic disorder (Nardi et al 2006), a personal history of anxiety, depression (Gregory et al 2007), and cardiac symptoms (Keyl and Eaton 1990). Since the variability in the predisposing and precipitating factors and comorbility in panic disorder is high, the nosology of panic disorder is still not perfect (Eaton et al 1995).

Research has found that more than $30 \%$ of patients with panic disorders have been diagnosed with mitral valve prolapse (MVP) (Liberthson et al 1986). A possible relationship is believed to exist between these two disorders, although research has shown inconsistent findings for such an association (Margraf et al 1988). According to Margraf and colleagues (1988), the reason for inconsistent results may be due to widely differing diagnostic criteria for MVP, lack of reliability of these criteria, and sampling bias in both case and controls. Some symptoms of MVP, such as chest oppression and palpitation, can evoke a panic attack. Hence, it is believed that panic disorder could be caused by MVP, or conversely, panic disorder may result in MVP (Pariser et al 1978; Gorman et al 1981; Filho et al 2008). Because the nosology of panic disorder is still not clear, it is possible that the association between panic disorder and MVP is coincidental.

Whether or not MVP patients are at higher risk for panic disorder and other psychiatric illnesses, and what percentage of MVP patients have experienced panic attack, are still uncertain (Tamam et al 2000). Therefore the aim of our study was to investigate the prevalence of panic disorder and other psychiatric disorders in young Han Chinese males with MVP. We controlled for age, sex, and ethnicity, and focused on the specific role MVP played in panic disorders.

\section{Methods}

\section{Participants}

Approval was first obtained from the institutional review board at a teaching hospital in southern Taiwan. Written informed consent was collected from all participants after a detailed explanation of the purpose of the study.
The participants were 18-25-year-old Taiwanese who came to the cardiac outpatient department with chest pain. Patients with congenital heart disease, cardiomyopathy, rheumatic heart disease, endocarditis, valve regurgitation, and hypertension were excluded. The echocardiograph was used by a senior cardiologist to diagnose MVP, and a total of 39 participants were recruited. All participants lived in a teaching hospital for at least one month to verify the diagnosis, and were interviewed by a senior psychiatrist, using the Mini-International Neuropsychiatric Interview (MINI) (Sheehan et al 1998).

\section{Materials}

This study used the Chinese version of the Mini-International Neuropsychiatric Interview (MINI) for the diagnosis of psychiatric disorders. The MINI was developed by Sheehan and colleagues (1998), and the Chinese version was translated by Lee and colleagues (unpublished data); this version has demonstrated good reliability and validity for diagnosing panic disorder.

\section{Statistical analysis}

Data were analyzed using SPSS 15.0 for Windows (SPSS Inc., Chicago, IL). All variables were analyzed using descriptive and chi-square analysis.

\section{Results \\ Demographic distribution and psychiatric prevalence}

Thirty-nine males between the ages of 18 and 25 participated in the study. Using echocardiographs, 12 (30.8\%) were diagnosed with MVP. The control group consisted of 27 (69.2\%) participants who had no abnormalities in the echocardiograph, but who complained of chest discomfort. The medical history and current symptoms of all participants met the diagnosis of at least one psychiatric disorder; 14 (35.9\%) met the diagnosis of anxiety disorder, and 18 (46.2\%) met at least one criterion for anxiety disorder. Nine (23.1\%) participants met the diagnostic criteria of major depressive disorder or other mood disorders.

\section{The association between MVP and panic disorder}

One (8.3\%) MVP patient and two (7.4\%) individuals in the control group met the diagnostic criteria of panic disorder at the time of the study. One (8.3\%) MVP patient and no individuals in the control group had a previous history of 
panic disorder. No statistically significant difference was found in the prevalence of panic disorder between the two groups.

\section{The association between MVP and other psychiatric disorders}

Using the MINI survey as the diagnostic tool for all psychiatric disorders, no statistically significant difference was found in the prevalence of psychiatric disorders between the MVP group and the control group.

\section{Discussion}

The results of our study showed that, among cardiac outpatient department individuals who reported chest discomfort, $35.9 \%$ of the patients met the diagnosis of anxiety disorder, $46.2 \%$ met at least one criterion of anxiety disorder, and $23.1 \%$ met the diagnostic criteria of major depressive disorder. This prevalence is higher than the $19.5 \%$ prevalence of anxiety disorder found by Kroenke and colleagues (2007) in a primary care setting. Some possible reasons, including ethnic and cultural differences, may contribute to the prevalence in the Chinese population. In general, Chinese tend to express somatically when coping with stress (Kleinman 1977; Parker et al 2001), and men especially tend to suffer from more frequent somatic complaints and anxiety (Tang and Lau 1996). The complaints could be related to socialcultural factors (Gackenbach 1982; Steer et al 1989), and to personality factors, which might have caused the higher prevalence in the present study.

Another reason might be the small sample, which is a limitation of our study. A possible type II error is our finding of a higher prevalence of psychiatric disorders in Chinese populations. Increasing the sample size of the subjects could help to confirm the prevalence of psychiatric disorders among MVP patients in further studies.

No statistically significant differences in the prevalence of panic disorder and other psychiatric disorders were found between the MVP and control groups, thereby demonstrating that MVP is not a risk factor for panic disorder. Patients with panic disorder are more likely to have cardiac abnormalities compared with controls (Venkatesh et al 1980), and the prevalence of MVP in panic disorder is high (Liberthson et al 1986). A previous study even proposed that MVP may play a role in the etiology of anxiety disorder (Hamada et al 1990). However, our study did not find that patients with MVP have a higher risk of panic disorder. Although the preliminary results of our study are limited by the sample size, all participants sought help at the cardiac outpatient department, and had symptoms of chest discomfort; we controlled for ethnic group, gender, and age. The onset age of panic disorder was found to peak at between 15 and 19 years in one study (Von Korff et al 1985). Our participants were all between the ages of 18 and 25 , and consequently the duration of illness for most of our subjects should not have been too long, thus reducing the chance for them to develop comorbid psychiatric and physical illnesses. Whether MVP may be a modifier or mediating factor for panic disorder needs to be further assessed in a larger scale study. In the present study, we also emphasize the need to establish adequate treatment for anxiety and mood disorders in primary care settings (Mechanic 2007), especially because other studies have indicated the under-diagnosis of psychiatric disorders in primary care settings (Kroenke et al 2007; Means-Christensen et al 2008). Furthermore, even those who have been diagnosed may not receive adequate treatment (Kroenke et al 2007; Weisberg et al 2007). This co-occurrence deserves attention in clinical practice, as well as attention to the prevalence of psychiatric disorders among patients suffering from MVP.

\section{Acknowledgments}

This study was supported by a grant (number 9306) from the Medical Affairs Bureau, Ministry of National Defense, Taiwan. The authors would also like to acknowledge all researchers who assisted in this study. The authors report no conflicts of interest in this work.

\section{References}

Andrade L, Eaton WW, Chilcaot HD. 1996. Lifetime co-morbidity of panic attacks and major depression in a population-based study: age of onset. Psychol Med, 26:991-6.

Bienvenu OJ, Onyike CU, Stein MB, et al. 2006. Agoraphobia in adults: incidence and longitudinal relationship with panic. Br J Psychiatry, 188:432-8.

Demertzis KH, Craske MG. 2006. Anxiety in primary care. Curr Psychiatry Rep, 8:291-7.

Domschke K, Deckert J, O’Donovan MC, et al. 2007. Meta-analysis of COMT val158met in panic disorder: ethnic heterogeneity and gender specificity. Am J Med Genet B, Neuropsychiatr Genet, 144:667-73.

Eaton WW, Badawi M, Melton B. 1995. Prodromes and precursors: epidemiologic data for primary prevention of disorders with slow onset. Am J Psychiatry, 152:967-72.

Filho AS, Maciel BC, Martin-Santos R, et al. 2008. Does the association between mitral valve prolapse and panic disorder really exist? Prim Care Companion J Clin Psychiatry, 10:38-47.

Freitag CM, Domschke K, Rothe C, et al. 2006. Interaction of serotonergic and noradrenergic gene variants in panic disorder. Psychiatr Genet, 16:59-65.

Fyer AJ, Hamilton SP, Durner M, et al. 2006. A third-pass genome scan in panic disorder: evidence for multiple susceptibility loci. Biol Psychiatry, 60:388-401.

Gackenbach J. 1982. Collegiate swimmers: sex differences in self-reports and indices of physiological stress. Percept Mot Skills, 55:555-8. 
Gorman JM, Fyer AF, Gliklich J, et al. 1981. Effect of imipramine on prolapsed mitral valves of patients with panic disorder. Am J Psychiatry, 138:977-8.

Gregory AM, Caspi A, Moffitt TE, et al. 2007. Juvenile mental health histories of adults with anxiety disorders. Am J Psychiatry, 164:301-8.

Hamada T, Fukui J, Koshino Y, et al. 1990. Mitral valve prolapse syndrome as an etiologic factor of anxiety disorder. Rinsho Byori, 38:952-6.

Keyl PM, Eaton WW. 1990. Risk factors for the onset of panic disorder and other panic attacks in a prospective, population-based study. Am J Epidemiol, 131:301-11.

Kleinman AM. 1977. Depression, somatization and the new cross-cultural psychiatry. Soc Sci Med, 11:3-10.

Kroenke K, Spitzer RL, Williams JB, et al. 2007. Anxiety disorders in primary care: prevalence, impairment, comorbidity, and detection. Ann Intern Med, 146:317-25.

Lang AJ, Norman GJ, Casmar PV. 2006. A randomized trial of a brief mental health intervention for primary care patients. $J$ Consult Clin Psychol, 76:1173-9.

Lecrubier Y. 2007. Widespread underrecognition and undertreatment of anxiety and mood disorders: results from 3 European studies. J Clin Psychiatry, 68(Suppl 2):36-41.

Liberthson R, Sheehan DV, King ME, et al. 1986. The prevalence of mitral valve prolapse in patients with panic disorders. Am J Psychiatry, 143:511-5.

Lieb R. 2005. Anxiety disorders: clinical presentation and epidemiology. Handb Exp Pharmacol, 169:405-32.

Margraf J, Ehlers A, Roth WT. 1988. Mitral valve prolapse and panic disorder: a review of their relationship. Psychosom Med, 50:93-113.

Means-Christensen AJ, Roy-Byrne PP, Sherbourne CD, et al. 2008. Relationships among pain, anxiety, and depression in primary care. Depress Anxiety, 25:593-600.

Mechanic D. 2007. Barriers to help-seeking, detection, and adequate treatment for anxiety and mood disorders: implications for health care policy. $J$ Clin Psychiatry, 68(Suppl 2):20-6.

Mergl R, Seidscheck I, Allgaier AK, et al. 2007. Depressive, anxiety and somatoform disorders in primary care: prevalence and recognition. Depress Anxiety, 24:185-95.

Messias E, Eaton W, Nestadt G, et al. 2007. Psychiatrists' ascertained treatment needs for mental disorders in a population-based sample. Psychiatr Serv, 58:373-7.

Nardi AE, Valenca AM, Mezzasalma MA, et al. 2006. Comparison between hyperventilation and breath-holding in panic disorder: patients responsive and non-responsive to both tests. Psychiatry Res, 142:201-8.
Pariser SF, Pinta ER, Jones BA. 1978. Mitral valve prolapse syndrome and anxiety neurosis/panic disorder. Am J Psychiatry, 135:246-7.

Parker G, Gladstone G, Chee KT. 2001. Depression in the planet's largest ethnic group: the Chinese. Am J Psychiatry, 158: 857-64.

Rothe C, Koszycki D, Bradwejn J, et al. 2006. Association of the Val158Met catechol O-methyltransferase genetic polymorphism with panic disorder. Neuropsychopharmacology, 31:2237-42.

Schur EA, Afari N, Furgerg H, et al. 2007. Feeling bad in more ways than one: comorbidity patterns of medically unexplained and psychiatric conditions. J Gen Intern Med, 2:818-21.

Sheehan DV, Lecrubier Y, Sheehan KH, et al. 1998. The Mini-International Neuropsychiatric Interview (M.I.N.I.): the development and validation of a structured diagnostic psychiatric interview for DSM-IV and ICD-10. J Clin Psychiatry, 59(Suppl 20):22-33.

Steer RA, Beck AT, Brown G. 1989. Sex differences on the revised Beck Depression Inventory for outpatients with affective disorders. $J$ Pers Assess, 53:693-702.

Swartz KL, Pratt LA, Armenian HK, et al. 2000. Mental disorders and the incidence of migraine headaches in a community sample: results from the Baltimore Epidemiologic Catchment area follow-up study. Arch Gen Psychiatry, 57:945-50.

Tamam L, Ozpoyraz N, San M, et al. 2000. Association between idiopathic mitral valve prolapse and panic disorder. Croat Med J, 41:410-6.

Tang CS, Lau BH. 1996. The Chinese Gender Role Stress scales. Factor structure and predictive validity. Behav Modif, 20:321-37.

Unschuld PG, Ising M, Erhardt A, et al. 2008. Polymorphisms in the galanin gene are associated with symptom-severity in female patients suffering from panic disorder. J Affect Disord, 105(1-3):177-84

Venkatesh A, Pauls DL, Crowe R, et al. 1980. Mitral valve prolapse in anxiety neurosis (panic disorder). Am Heart $J, 100: 302-5$.

Von Korff MR, Eaton WW, Keyl PM. 1985. The epidemiology of panic attacks and panic disorder. Results of three community surveys. Am J Epidemiol, 122:970-81.

Weisberg RB, Dyck I, Culpepper L, et al. 2007. Psychiatric treatment in primary care patients with anxiety disorders: a comparison of care received from primary care providers and psychiatrists. Am J Psychiatry, 164:276-82. 